\title{
Resistance to spiramycin in Streptomyces ambofaciens, the producer organism, involves at least two different mechanisms
}

\author{
Jean-Luc Pernodet, ${ }^{*}$ Maria-Teresa Alegre, $\dagger$ Marie-Hélène Blondelet-Rouault and \\ MiCHEL GUÉRINEAU
}

Institut de Génétique et de Microbiologie, URA CNRS 1354, Bâtiment 400, Université Paris XI, 91405 Orsay Cedex, France

(Received 29 September 1992; revised 21 December 1992; accepted 7 January 1993)

\begin{abstract}
During its stationary phase, Streptomyces ambofaciens produces the macrolide antibiotic spiramycin, and has to protect itself against this antibiotic. Young mycelia, not yet producing spiramycin, are sensitive to it, but they become fully resistant when production begins. In a sensitive mycelium, resistance could be induced by exposure to sub-inhibitory concentrations of spiramycin, and these induced mycelia, like producing mycelia were resistant not only to spiramycin but also to several other macrolide antibiotics. Ribosomes extracted from these resistant mycelia were shown in vitro to be more resistant to spiramycin than ribosomes extracted from sensitive mycelium, indicating that $S$. ambofaciens possesses a spiramycin-inducible ribosomal resistance to spiramycin and to macrolide antibiotics. Studies with spiramycin non-producing mutants showed that, in these mutants, resistance to spiramycin also varies during cultivation, in that an old culture was much more resistant than a young one. But with these non-producing mutants, the spectrum of resistance was narrower, and in vitro data showed that resistance was not due to ribosomal modification. These results suggest that $S$. ambofaciens presents at least two distinct mechanisms for spiramycin resistance; a spiramycin-inducible ribosomal resistance, and a second resistance mechanism which might be temporally regulated and which could involve decreased permeability to, or export of, the antibiotic. The two mechanisms are probably at work simultaneously in the producing mycelium, the spiramycin-inducible resistance being induced by endogenous spiramycin. In non-producing mutants, in the absence of self-induction by spiramycin, only the second mechanism is observed.
\end{abstract}

\section{Introduction}

Macrolide antibiotics are compounds consisting of a macrocyclic lactone ring of 12,14 or 16 residues to which are attached one or more amino or deoxy sugars (Omura \& Tanaka, 1984). These antibiotics inhibit protein synthesis in prokaryotes by a mechanism involving binding of the antibiotic to the 50S ribosomal subunit (Gale et al., 1981). Macrolides, especially erythromycin, have been widely used in human medicine. Several mechanisms of resistance to erythromycin or to macrolides have been described. Mutations affecting the genes encoding ribosomal components can confer resistance. For instance, in Escherichia coli, mutants conferring

*Author for correspondence. Tel. 33169416913 ; fax 331694172 96.

$\dagger$ Present address: Escuela Universitaria Ingeniria Técnica Agricola, 81 Avenida de Madrid, 27002 Lugo, Spain. erythromycin resistance have been located in the genes coding for ribosomal proteins L4 and L22 (Wittmann et al., 1973) and macrolide resistance mutations have been obtained in the 23S rRNA genes (Morgan et al., 1988). Resistance to erythromycin by enzymic hydrolysis of the drug has also been reported in E. coli (Barthélémy et al., 1984). Active efflux of the drug, mediated by a protein of the ATP-binding transport family, is responsible for the resistance observed in some strains of staphylococci (Ross et al., 1990). However, in pathogenic bacteria, the most widespread resistance is known as MLS resistance because it confers resistance to macrolide, lincosamide and streptogramin type $B$ antibiotics (reviewed by Weisblum, 1983; Leclercq \& Courvalin, 1991). Here resistance is due to a modification of the $50 \mathrm{~S}$ ribosomal subunit by methylation of an adenine residue in the $23 \mathrm{~S}$ rRNA. This MLS-resistant phenotype can be expressed constitutively or can be induced by sub-inhibitory concentrations of some MLS antibiotics, generally including erythromycin. 
Several Streptomyces species are macrolide producers, but among Streptomyces species, whether producers of MLS antibiotics or not, some present the archetypal MLS resistance phenotype and others have only partial MLS resistance, suggesting that a family of macrolide and lincosamide resistance mechanisms is distributed among these species (Fujisawa \& Weisblum, 1981). Genes encoding 23S rRNA methyl transferases conferring resistance have been isolated from several streptomycete strains and characterized (reviewed by Cundliffe, $1989 a, b$ ). Other resistance mechanisms have also been reported in Streptomyces. For instance, Streptomyces coelicolor Müller, which is not known to be a macrolide producer, can nevertheless $O$-phosphorylate macrolides (Marshall et al., 1989). Streptomyces antibioticus, the oleandomycin producer, has been shown to have a decreased permeability to oleandomycin, suggesting a role for cell permeability in self-resistance (Fierro et al., 1987), and a macrolide glycosyl-transferase activity has been characterized in the same strain (Vilches et al., 1992). A macrolide glycosyl-transferase is also present in Streptomyces lividans, and the gene encoding this enzyme has been isolated and sequenced (Jenkins \& Cundliffe, 1991). Streptomyces vendargensis can also inactivate erythromycin by glycosylation (Kuo et al., 1989). Macrolide producers very often have several different genes involved in self resistance. For instance, Streptomyces fradiae, the tylosin producer, has had four different resistance genes cloned from it (Birmingham et al., 1986; Baltz \& Seno, 1988; Beckmann et al., 1989; Zalacain \& Cundliffe, 1991).

Streptomyces ambofaciens produces spiramycin (Pinnert-Sindico, 1954), a 16-membered macrolide containing three amino sugars. In this organism, a mutation in one of the 23S ribosomal RNA genes confers a higher level of resistance to macrolide antibiotics (Pernodet et al., 1988). We report here the results of a physiological study of spiramycin resistance in S. ambofaciens.

\section{Methods}

Bacterial strains, media and culture conditions. The wild-type $S$. ambofaciens strain (ATCC23877), and three $S$. ambofaciens spiramycin non-producing mutants (RP17, RP167, NP3) were used. Mutants RP17 and RP167 were obtained after NTG mutagenesis and were a kind gift of Dr P. Lacroix, Rhone-Poulenc Rorer, Vitry, France. Mutant NP3 was obtained after mutagenesis by nitrous acid (Smokvina et al., 1988) and was a kind gift of Dr F.-X. Francou, Université Paris XI, Orsay, France.

S. ambofaciens strains were maintained on plates of Hickey-Tressner (HT) medium (Pridham et al., 1957) at $30^{\circ} \mathrm{C}$. This medium contained (per litre) $1 \mathrm{~g}$ yeast extract (Difco), $1 \mathrm{~g}$ beef extract (Difco), $10 \mathrm{~g}$ white dextrin (Prolabo), $2 \mathrm{~g}$ NZ amine type A (Sheffield), $20 \mathrm{mg}$ $\mathrm{CoCl}_{2} .7 \mathrm{H}_{2} \mathrm{O}, 20 \mathrm{~g}$ Bacto agar (Difco). The $\mathrm{pH}$ was adjusted to $7 \cdot 3$ prior to autoclaving. Spores harvested from HT plates were separated from mycelial fragment by filtration on cotton, suspended in $20 \%$ (w/v) glycerol and stored at $-20^{\circ} \mathrm{C}$ until used. YEME liquid medium (Bibb et al., 1977) contained (per litre) $3 \mathrm{~g}$ yeast extract (Difco), $5 \mathrm{~g}$ Bacto-peptone (Difco), $3 \mathrm{~g}$ malt extract (Difco), $10 \mathrm{~g}$ glucose, $340 \mathrm{~g}$ sucrose. After autoclaving, $\mathrm{MgCl}_{2}$ was added to a final concentration of $5 \mathrm{~mm}$, and glycine to a final concentration of $0.25 \%$. DALP liquid medium (Dr A. Sabatier, personal communication) contained (per litre) $10 \mathrm{~g}$ Bacto-peptone (Difco), $5 \mathrm{~g}$ yeast extract (Difco), $10 \mathrm{~g}$ white dextrin (Prolabo). Prior to autoclaving, the $\mathrm{pH}$ was adjusted to 7.0 and Bacto agar (Difco) was added to the medium to a final concentration of $0.2 \mathrm{~g}^{-1}$. These media were inoculated with spores and incubated at $30^{\circ} \mathrm{C}$ on an orbital shaker. For spiramycin production, two different media were used. The first, SL11 (Dr A. Sabatier, personal communication), contained (per litre) $25 \mathrm{~g}$ white dextrin (Prolabo), $12.5 \mathrm{~g}$ yeast extract (Fould-Springer), $1 \mathrm{~g} \mathrm{MgSO}_{4}, 1 \mathrm{~g} \mathrm{KH}_{2} \mathrm{PO}_{4}, 20.9 \mathrm{~g}$ MOPS (Sigma). The $\mathrm{pH}$ was adjusted to $7 \cdot 1$ and the medium was put into Erlenmeyer flasks. Prior to autoclaving, $\mathrm{CaCO}_{3}$ was added to the medium to a final concentration of $5 \mathrm{~g} \mathrm{l}^{-1}$, and maize oil to a final concentration of $0.5 \%$. SL11 medium was inoculated with spores. The second production medium, MP5, contained (per litre) $7 \mathrm{~g}$ yeast extract (Difco), $5 \mathrm{~g} \mathrm{NaCl}, 1 \mathrm{~g} \mathrm{NaNO}, 36 \mathrm{ml}$ glycerol, $20.9 \mathrm{~g} \mathrm{MOPS}$ (Sigma). The $\mathrm{pH}$ was adjusted to 7.5 prior to autoclaving. As germination was not very good in MP5, this medium was inoculated with spores incubated for $2 \mathrm{~h}$ at $37^{\circ} \mathrm{C}$ in pre-germination medium as described (Hopwood et al., 1985). For spiramycin production, the cultures $(60 \mathrm{ml}$ in a $500 \mathrm{ml}$ flask) were incubated at $26^{\circ} \mathrm{C}$ in an orbital shaker at 260 r.p.m. SL11 was the better medium for production, but the insoluble $\mathrm{CaCO}_{3}$ present in this medium was an obstacle when ribosomes had to be prepared from cultures grown in production medium. In such cases, MP5 was used.

Antibiotic production and resistance. Antibiotic production was tested by a biological assay. Bacillus subtilis ATCC6633 was used as the indicator organism. A paper disk was impregnated with $80 \mu \mathrm{l}$ Streptomyces culture supernatant, and was laid on the surface of an agar plate spread with a spore suspension of the indicator organism. The medium was antibiotic medium no. 8 (Difco) buffered with $100 \mathrm{~mm}$-phosphate buffer, $\mathrm{pH} \mathrm{8.0.} \mathrm{After} \mathrm{overnight} \mathrm{incubation} \mathrm{at} 37^{\circ} \mathrm{C}$, the diameter of the inhibition zone was measured. As standards, disks impregnated with various dilutions of a solution of commercial spiramycin were also laid on the surface of the plate. The diameters of the inhibition zones for these disks were used to draw the standard curve. The threshold of detection for this method was $0.5 \mu \mathrm{g}$ spiramycin $\mathrm{ml}^{-1}$ in the production medium.

To assay mycelial spiramycin resistance, Streptomyces strains were grown in liquid medium, in Erlenmeyer flasks. Samples were periodically withdrawn and gently sonicated (for $20 \%$ of a period of $2 \mathrm{~min}$, at $40 \mathrm{~W}$ with a Branson 250 sonicator) to disperse the mycelial pellets, diluted and plated on medium without spiramycin to give the total colony-forming units (c.f.u.), and on medium with spiramycin to give resistant c.f.u. Colonies were counted after $3 \mathrm{~d}$ incubation.

Assay of in vivo protein synthesis. S. ambofaciens was grown in the production medium. When necessary, resistance was induced by exposure to exogenous spiramycin for $2 \mathrm{~h}$. The mycelium was centrifuged and resuspended in one third of the culture volume of minimal medium (Fierro et al., 1987) containing (per litre) $10 \mathrm{~g}$ glucose, $2 \mathrm{~g}$ asparagine, $2 \mathrm{~g}\left(\mathrm{NH}_{4}\right)_{2} \mathrm{SO}_{4}, 4.3 \mathrm{~g} \mathrm{~K}_{2} \mathrm{HPO}_{4}, 0.82 \mathrm{~g} \mathrm{KH}_{2} \mathrm{PO}_{4}, 0.01 \mathrm{~g}$ $\mathrm{FeSO}_{4} .7 \mathrm{H}_{2} \mathrm{O}, 0.5 \mathrm{~g} \quad \mathrm{MgSO}_{4} \cdot 7 \mathrm{H}_{2} \mathrm{O}$. The mycelium was then homogenized with a Thoma homogenizer. Samples of $300 \mu$ were incubated at $25^{\circ} \mathrm{C}$ for $15 \mathrm{~min}$ in the absence or presence of various concentrations of antibiotics, followed by pulse-labelling for $30 \mathrm{~min}$ with $2.5 \mu \mathrm{Ci}$ of $\left.{ }^{35} \mathrm{~S}\right]$ methionine $\left(1123 \mathrm{Ci} \mathrm{mmol}^{-1}, 41 \cdot 5 \mathrm{MBq} \mathrm{mmol}^{-1}\right)$.

We then added $50 \mu \mathrm{l}$ cold methionine $(250 \mathrm{~mm})$ and incubated at $25^{\circ} \mathrm{C}$ for $10 \mathrm{~min}$ more. Incorporation was stopped by the addition of $0.8 \mathrm{ml} 20 \%$ (w/v) TCA. After $10 \mathrm{~min}$ on ice, the samples were heated at $90^{\circ} \mathrm{C}$ for $15 \mathrm{~min}$, cooled on ice and filtered through Whatman GF/C 
filters. The radioactivity in the dried filters was measured by liquidscintillation counting, using a toluene-based scintillation fluid.

Preparation of ribosomes and post-ribosomal supernatant. Ribosomes and post-ribosomal supernatants (S100*) were prepared from Streptomyces ambofaciens as described by Skeggs et al. (1985), except that the mycelium was ground with alumina in a mortar instead of being passed through a French press.

Poly-U-directed polyphenylalanine synthesis. This was carried out essentially as described by Skeggs et al. (1985). For some experiments, a post-ribosomal supernatant from Escherichia coli, called S105, was used instead of $5100^{*}$ from Streptomyces ambofaciens. This postribosomal supernatant was a kind gift from J. Dondon, Institut de Biologie Physico-Chimique, Paris France.

\section{Results}

Changes in the resistance level to spiramycin during cultivation

Under our culture conditions, in the production media (SL11 and MP5), spiramycin production begins after $36 \mathrm{~h}$ cultivation (see below). The sensitivity of young mycelium, not yet producing after $24 \mathrm{~h}$ cultivation, and of mycelium producing spiramycin after $72 \mathrm{~h}$ of growth, were assayed. The results (Fig. 1) show clearly that old antibiotic-producing mycelium is much more resistant to spiramycin than mycelium which is not producing yet. For instance, when plated on medium containing $400 \mu \mathrm{g}$ spiramycin $\mathrm{ml}^{-1}, 100 \%$ of the c.f.u. from the producing culture grew, compared to $0.1 \%$ of the c.f.u. from the young culture. This concentration of $400 \mu \mathrm{g}$ spiramycin $\mathrm{ml}^{-1}$ was used throughout this study to discriminate between sensitive and resistant mycelium. Resistance was expressed as the ratio of the c.f.u. on medium with $400 \mu \mathrm{g}$ spiramycin $\mathrm{ml}^{-1}$ to the c.f.u. on medium without spiramycin. Thus, the resistance of producing mycelium was $100 \%$. To know more precisely when the change in the resistance level of the culture occurred, mycelium grown in production medium was assayed for resistance at regular intervals throughout cultivation. The concentration of spiramycin in the supernatant was also determined, using a biological assay. The results (Fig. 2) were obtained in SL11 production medium, but identical results were obtained with MP5. The medium was inoculated with spores which are relatively resistant to spiramycin, but as they germinated they became spiramycin-sensitive, and resistance was minimal after $24 \mathrm{~h}$ growth, after which time no spiramycin could be detected in the medium. The resistance then increased very rapidly, and the mycelium was fully resistant after $36 \mathrm{~h}$ growth. Spiramycin could be detected in the medium at this time, although it could have been present in small amounts inside the mycelium before it could be detected in the supernatant. Thus the increase in the resistance level and the start of production

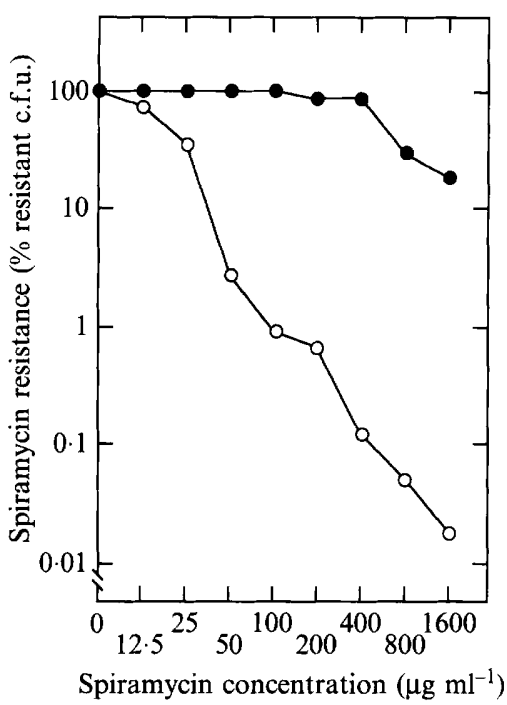

Fig. 1. Sensitivity of $S$. ambofaciens to spiramycin. The mycelium was gently sonicated to disrupt the pellets and dilutions were plated on medium either without spiramycin or with various concentrations of the drug. Survival is expressed as the ratio c.f.u. on medium with spiramycin to c.f.u. on the same medium without spiramycin. $O, 24 \mathrm{~h}$ culture of $S$. ambofaciens, before production phase; $72 \mathrm{~h}$ culture of $S$. ambofaciens during production phase.

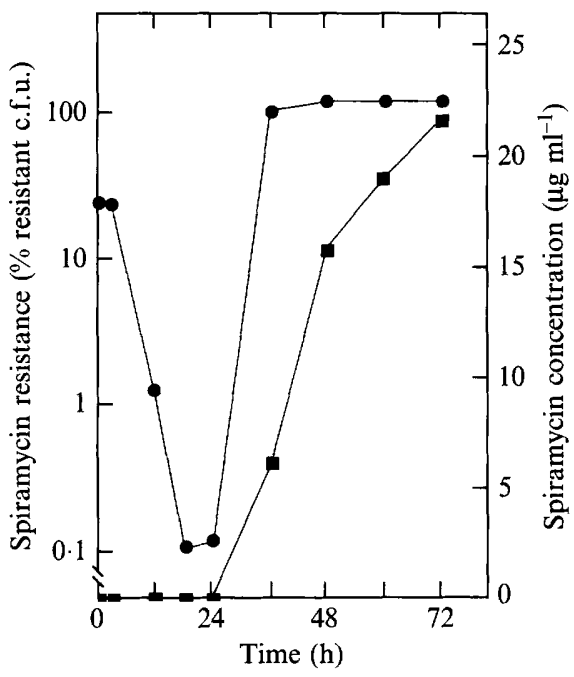

Fig. 2. Spiramycin resistance and production during growth of $S$. ambofaciens ATCC23877. S. ambofaciens was grown in production medium. At regular time intervals, samples were taken of the culture. The mycelium was gently sonicated to disrupt the pellets, and spiramycin resistance $(\boldsymbol{O})$ and production ( $\boldsymbol{\square})$ were assayed as described in Methods. Resistance is expressed as the ratio c.f.u. on medium with spiramycin $\left(400 \mu \mathrm{g} \mathrm{ml}^{-1}\right)$ to c.f.u. on the same medium without spiramycin.

may well be concomitant. MLS resistance can be induced by some antibiotics of the MLS group in several other bacteria. It is possible that in $S$. ambofaciens sub- 
inhibitory concentrations of spiramycin present inside the mycelium induce a resistance mechanism of the MLS type.

\section{Induction of spiramycin resistance by sub-inhibitory concentrations of spiramycin}

In YEME medium, spiramycin production is not only reduced compared to that obtained in SL11 or MP5, but also delayed, because production starts after 3 or $4 \mathrm{~d}$ culture. In YEME medium, the mycelium remained very
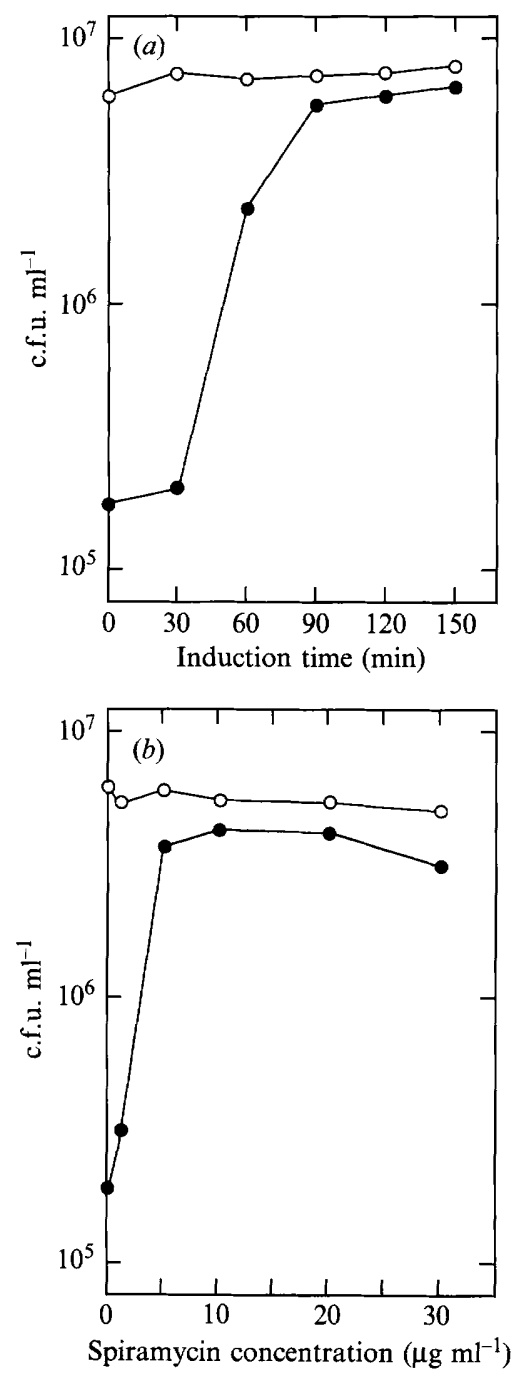

Fig. 3. Induction of spiramycin resistance by sub-inhibitory concentrations of spiramycin. S. ambofaciens was grown for $24 \mathrm{~h}$ in YEME medium. Spiramycin was added at time 0 . Samples were removed and dilutions were plated to measure c.f.u. on medium without spiramycin (O) or with $400 \mu \mathrm{g}$ spiramycin $\mathrm{ml}^{-1}(\bigcirc)$. (a) Time course of induction. The mycelium was exposed to a concentration of $10 \mu \mathrm{g}$ spiramycin ml $\mathrm{I}^{-1}$ and samples were removed at regular time intervals. $(b)$ Dose-response relationship. The mycelium was exposed for $2 \mathrm{~h}$ to various concentrations of spiramycin. sensitive to spiramycin during the period between 18 and $72 \mathrm{~h}$ culture (data not shown). Induction experiments using exogenous spiramycin were therefore performed in YEME medium rather than production medium, to avoid self-induction by endogenous spiramycin. A spiramycin-sensitive mycelium, grown in YEME liquid medium for $18 \mathrm{~h}$, was exposed to sub-inhibitory concentrations of spiramycin. Sub-inhibitory concentrations were defined as those antibiotic concentrations which did not affect the growth rate of the strain. The results (Fig. $3 a$ ) show that after $2 \mathrm{~h}$ exposure to $10 \mu \mathrm{g}$ spiramycin $\mathrm{ml}^{-1}$, the mycelium became spiramycinresistant, whereas the control (same mycelium but not exposed to spiramycin) remained fully sensitive (data not shown). Moreover, Fig. 3(b), shows both that the response was dependent on the spiramycin concentration, and that the spiramycin concentrations used were sub-inhibitory, as c.f.u. were not lowered on medium without spiramycin.

In the same way it was also possible to induce resistance in a mycelium grown in DALP medium or in the production media SL11 and MP5. The kinetics of induction were the same, but spiramycin concentrations as low as $1 \mu \mathrm{g} \mathrm{ml}^{-1}$ were sufficient to obtain full induction in production media (data not shown).

To investigate whether the resistance observed after induction by spiramycin was restricted to spiramycin or could be extended to other macrolide antibiotics, simple tests of alteration of the inhibition zone were performed as described by Fujisawa \& Weisblum (1981). These tests showed that spiramycin could induce resistance to other antibiotics such as chalcomycin or erythromycin to which the strain is otherwise very sensitive (data not shown). These results were confirmed by the sensitivity of protein synthesis to macrolide antibiotics (see below).

Thus sub-inhibitory concentrations of spiramycin can induce resistance to spiramycin and to other macrolide antibiotics. This resistance, which is induced by exogenous spiramycin, should also be induced by endogenous spiramycin when production of the antibiotic begins. We wondered whether this resistance mechanism was the only one responsible for the resistant phenotype observed after $3 \mathrm{~d}$ cultivation in production medium, and if spiramycin was the only inducer. If so, this would imply that non-producing mutants should always remain sensitive to spiramycin. To test this hypothesis, spiramycin-non-producing mutants were studied.

\section{Study of non-producing mutants}

The spiramycin-non-producing mutants used in this study were blocked mutants capable of cosynthesis ( $P$. Lacroix and F.-X. Francou, personal communication), i.e. when two different mutants were grown nearby on an 


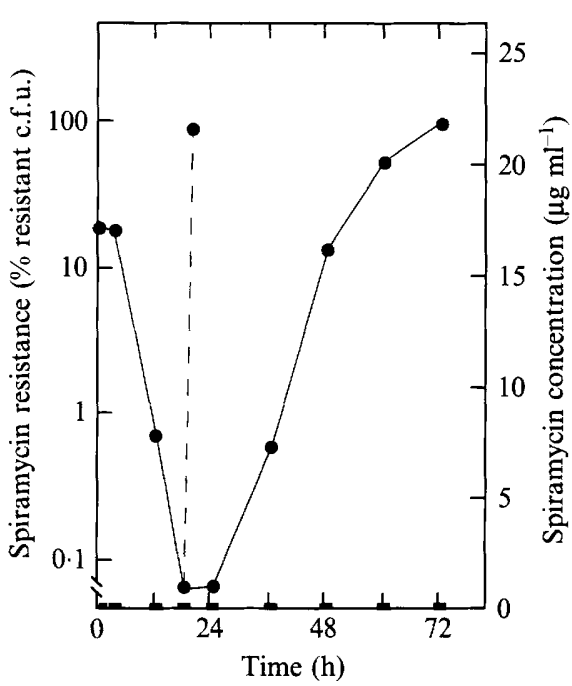

Fig. 4. Spiramycin resistance during growth of a non-producing mutant of $S$. ambofaciens. The non-producing mutant was grown and resistance was followed as described for the wild-type strain. No spiramycin ( $\square$ ) could be detected in the culture supernatant. The resistance (O) is expressed as the ratio of c.f.u. on medium with spiramycin $\left(400 \mu \mathrm{g} \mathrm{ml}^{-1}\right)$ to c.f.u. on the same medium without spiramycin. The dotted line represents induction of resistance when exogenous spiramycin was added to a final concentration of $1 \mu \mathrm{g} \mathrm{ml}^{-1}$ to a sample of the $18 \mathrm{~h}$ culture.

agar plate, a spiramycin biosynthetic intermediate accumulated by one of the mutants was secreted and then taken up and converted to spiramycin by the other mutant, blocked at an earlier step of the pathway. This means that the mutants are probably affected in one or a few genes of the spiramycin biosynthetic pathway, and that they are blocked in different steps of the pathway.

The resistance of these non-producing mutants was followed during their cultivation in production media SL11 or MP5, as described above for the wild-type strain. The results shown (Fig. 4) were obtained with mutant RP17 grown in SL11, but identical results were obtained with the other non-producing mutants. From these results, it is clear that even with non-producing mutants, there is an increase in the resistance level of the strain, beginning after $24 \mathrm{~h}$ growth and yielding a mycelium fully resistant to $400 \mu \mathrm{g}$ spiramycin $\mathrm{ml}^{-1}$. The increase in the resistance level is less rapid with nonproducing mutants than with the wild-type strain (compare Figs 2 and 4).

With all non-producing mutants used, it was possible to induce the resistance of a sensitive mycelium, after $18 \mathrm{~h}$ cultivation, by exposure to sub-inhibitory spiramycin concentrations. The parameters of induction were the same as for the wild-type strain, and the mycelium also became more resistant to macrolide antibiotics. This induction by exogenous spiramycin is shown in Fig. 4. The spiramycin-inducible resistance mechanism present in the wild-type strain is also functional in the non-producing mutants.

To explain the resistance observed in the nonproducing mutants after $72 \mathrm{~h}$ cultivation, without induction by exogenous spiramycin, two hypotheses can be advanced. In the first hypothesis, the resistance observed after $72 \mathrm{~h}$ cultivation occurs by the same mechanism as that responsible for the spiramycin-inducible resistance. This mechanism would be active either because the so called 'non-producing mutants' are nevertheless producing undetected spiramycin in amounts sufficient to induce resistance, or because it could respond to an inducer other than spiramycin, perhaps a spiramycin precursor in the biosynthetic pathway, although if so, such a precursor must be the product of an early step in the pathway, prior to the steps in which the mutants are blocked, because different mutants behave in the same way. If the same mechanism is responsible for both the resistance observed after induction by exogenous spiramycin and that observed after $72 \mathrm{~h}$ of cultivation, then the spectrum of resistance should be the same in each. In the second hypothesis, the resistance observed after $72 \mathrm{~h}$ cultivation occurs by a second mechanism, different to that involved in the spiramycin-inducible resistance. This second mechanism could differ in its spectrum from that first identified. Thus the next step was to study the spectrum of resistance in the wild-type strain and in the non-producing mutants after either induction by exogenous spiramycin or $72 \mathrm{~h}$ cultivation.

\section{Susceptibility of protein synthesis in vivo}

The aim of these experiments was to determine the spectrum of resistance of the mycelia of the wild-type strain and of the non-producing mutants, after $24 \mathrm{~h}$ cultivation, with or without induction by exogenous spiramycin, and after $72 \mathrm{~h}$ cultivation without addition of exogenous spiramycin. To determine the spectrum of resistance accurately, the susceptibility of in vitro protein synthesis was studied by a technique in which the mycelium was incubated in the absence or presence of various macrolide antibiotics for $10 \mathrm{~min}$, followed by pulse-labelling with $\left[{ }^{35} \mathrm{~S}\right]$ methionine for $30 \mathrm{~min}$. The mycelium was exposed to the macrolide antibiotics for too short a time to allow induction of resistance by these antibiotics. Fig. 5 shows results from one typical experiment. Similar results were reproducibly obtained with mycelia from different cultures grown in the same conditions. For the wild type strain, mycelium induced by exogenous spiramycin, and producing mycelium, are both more resistant to several macrolide antibiotics than the uninduced non-producing mycelium. In the case of the non-producing mutants, induction by exogenous spiramycin conferred the same spectrum of resistance to 


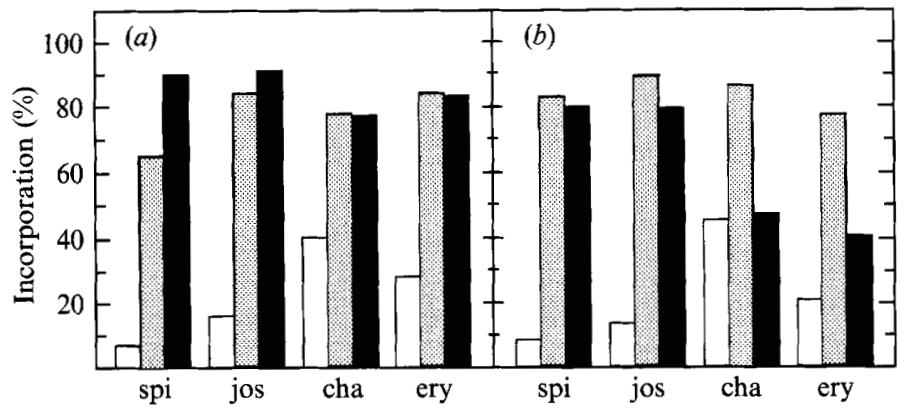

Fig. 5. Effect of macrolides on protein synthesis by whole cells. The activity is the ratio of $\left[{ }^{35} \mathrm{~S}\right] \mathrm{methionine}$ incorporation in the presence of various macrolide antibiotics compared to the incorporation in the absence of antibiotic: spi, $10 \mu \mathrm{g} \mathrm{spiramycin} \mathrm{ml}^{-1}$; jos, $1 \mu \mathrm{g}$ josamycin $\mathrm{ml}^{-1}$; cha, $0.5 \mu \mathrm{g}$ chalcomycin $\mathrm{ml}^{-1}$; ery, $0.5 \mu \mathrm{g}$ erythromycin $\mathrm{ml}^{-1}$. (a) Mycelium of the wild-type $S$. ambofaciens strain from: $\square$, one-day-old culture, non-induced (sensitive mycelium) (100\% activity: 12732 c.p.m.); 圆, one-day-old culture, induced (resistant mycelium) (100\% activity: 8030 c.p.m.); $\mathbf{\square}$, three-day-old culture (resistant mycelium) (100\% activity: 14402 c.p.m.). (b) Mycelium of the spiramycin non-producing mutant from: $\square$, one-day-old culture, non-induced (sensitive mycelium) (100\% activity: 11424 c.p.m.); 圈, one-day-old culture, induced (resistant mycelium) (100\% activity: 10928 c.p.m.); $\boldsymbol{\square}$, three-day-old culture (resistant mycelium) (100\% activity: 21463 c.p.m.).

macrolide antibiotics as in the wild-type strain. But 3-dold mycelium of the non-producing mutants, which is spiramycin-resistant, was less resistant to erythromycin or chalcomycin than young mycelium induced by exogenous spiramycin. Since spectrum of resistance of the 3-d-old mycelium was narrower, the mechanism responsible for spiramycin resistance in old cultures of the non-producing mutant may well be different from that in the spiramycin-inducible resistance to macrolide antibiotics.

\section{Susceptibility of ribosomes to spiramycin}

Study of the biochemical basis of the resistance could confirm that two different mechanisms produce the two resistance types observed. The spiramycin-inducible mechanism seems to confer resistance to macrolide antibiotics, and this type of wide-spectrum resistance could be due to a modification of the target, i.e. the ribosome. Accordingly, we studied the effect of spiramycin on poly-U-directed synthesis of polyphenylalanine. Salt-washed ribosomes and postribosomal supernatant $\left(\mathrm{S} 100^{*}\right)$ were prepared from $24 \mathrm{~h}$ cultures of the wild-type strain grown in DALP medium, induced or not induced by exogenous spiramycin. Ribosomes from each were supplemented with both types of $\mathrm{S} 100^{*}$ and assayed in cell-free protein synthesizing systems directed by poly- $U$, in the absence or presence of various spiramycin concentrations. The results (Fig. 6) show that ribosomes from the induced culture are more resistant to spiramycin than those from the non-induced culture, regardless of the origin of the S100* fraction. Thus ribosome modification seems to be the basis of the resistance observed after spiramycin induction. This was confirmed by poly-U-directed

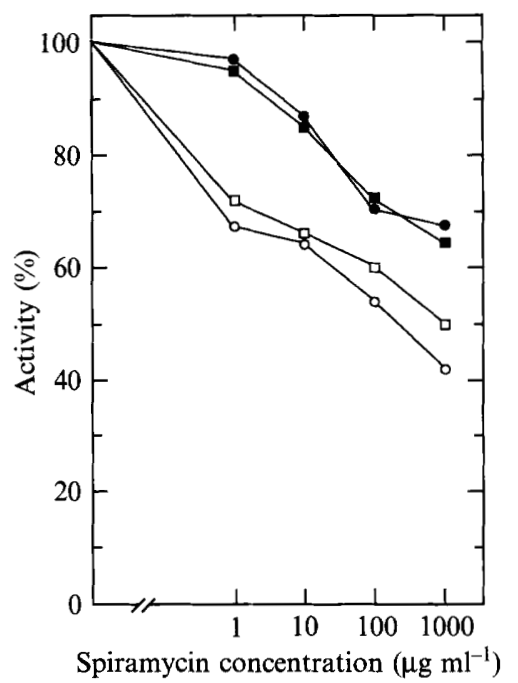

Fig. 6. Effect of spiramycin on poly-U-directed polyphenylalanine synthesis in vitro. Assays contained $\mathrm{S} 100^{*}$ and ribosomes from induced or non-induced S. ambofaciens mycelium: $\bullet$, ribosomes and $S 100^{*}$ from induced mycelium (100\% activity: 6237 c.p.m.); $\bigcirc$, ribosomes and S100* from non-induced mycelium ( $100 \%$ activity: 20690 c.p.m.); a, ribosomes from induced mycelium and $\mathrm{S} 100^{*}$ from non-induced mycelium (100\% activity: 9817 c.p.m.); $\square$, ribosomes from noninduced mycelium and $\mathrm{S} 100$ ) from induced mycelium (100\% activity: 15054 c.p.m.).

polyphenylalanine synthesis experiments using ribosomes from induced and non-induced cultures and S105 from $E$. coli (data not shown).

The next step was to compare the spiramycin susceptibility of ribosomes from $72 \mathrm{~h}$ cultures with that of ribosomes from $24 \mathrm{~h}$ induced and non-induced cultures, using the wild-type strain and non-producing mutants grown in MP5 medium. In all these experiments, S105 fractions from $E$. coli were used. The data shown 


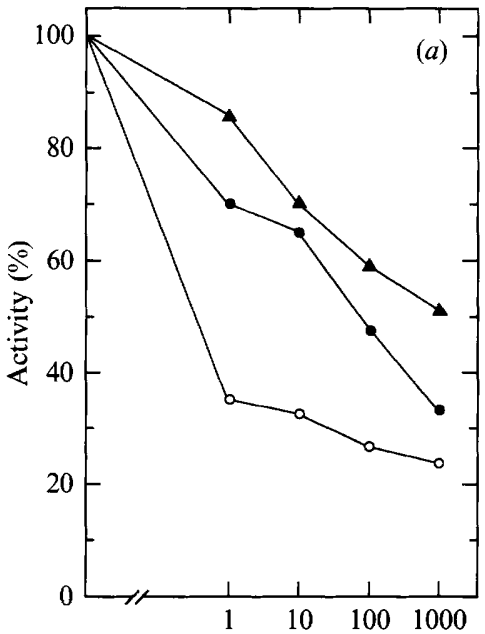

Spiramycin concentration $\left(\mu \mathrm{g} \mathrm{ml}^{-1}\right)$

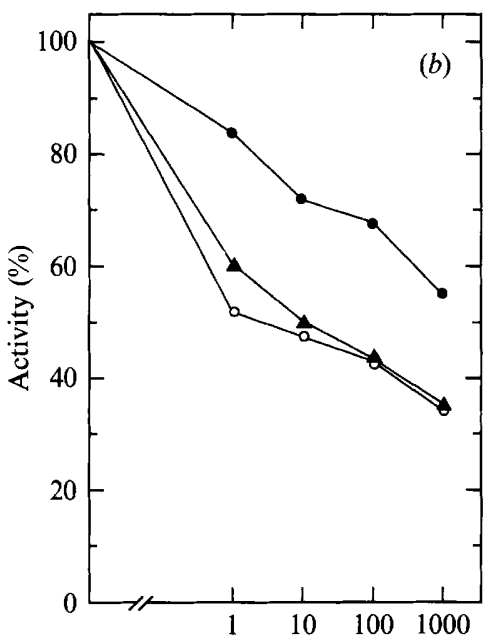

Spiramycin concentration $\left(\mu \mathrm{g} \mathrm{ml}^{-1}\right)$

Fig. 7. Effect of spiramycin on poly-U-directed polyphenylalanine synthesis in vitro. Assays contained $\mathrm{S} 105$ from $E$. coli and $S$. ambofaciens ribosomes. (a) Ribosomes from wild-type $S$. ambofaciens strain were extracted from: $O$, one-day-old culture, non-induced (sensitive mycelium) (100\% activity: 40176 c.p.m.); 9 , one-day-old culture, induced (resistant mycelium) (100\% activity: 20480 c.p.m.); $\Delta$, three-day-old culture (resistant mycelium) $(100 \%$ activity: 41165 c.p.m.). (b) Ribosomes from the spiramycin-non-producing mutant were extracted from: $O$, one-day-old culture, non-induced (sensitive mycelium) (100\% activity: 17893 c.p.m.); culture, induced (resistant mycelium) (100\% activity: 37423 c.p.m.); $\boldsymbol{\Delta}$, three-day-old culture (resistant mycelium) (100\% activity: 27840 c.p.m.).

(Fig. 7) were obtained with strain RP17, but the different non-producing mutants gave equivalent results. From Fig. $7(a)$, it is clear that the ribosomes from both the $72 \mathrm{~h}$ producing culture (resistant mycelium) and the $24 \mathrm{~h}$ induced culture (resistant mycelium) are more resistant to spiramycin than the one from the $24 \mathrm{~h}$ uninduced culture (sensitive mycelium). The difference observed between the ribosomes from the $24 \mathrm{~h}$ induced culture and those from the $72 \mathrm{~h}$ producing culture might be due to incomplete modification of the ribosomes after only $2 \mathrm{~h}$ induction, and the fact that the ribosome population tested is not homogeneous. In the case of the spiramycinnon-producing mutant (Fig. $7 \mathrm{~b}$ ), only ribosomes from the $24 \mathrm{~h}$ induced culture (resistant mycelium) display resistance to spiramycin. Ribosomes from the $72 \mathrm{~h}$ culture (resistant mycelium) are as sensitive to spiramycin as ribosomes from the $24 \mathrm{~h}$ non-induced culture (sensitive mycelium). Ribosomal modification is therefore responsible for the resistant phenotype observed after induction either by exogenous spiramycin added to $24 \mathrm{~h}$ culture or by endogenous spiramycin produced by the wild-type strain after $72 \mathrm{~h}$ cultivation. But ribosomal modification cannot account for the resistance observed in $72 \mathrm{~h}$ cultures of the non-producing mutants, demonstrating that another mechanism is involved.

\section{Discussion}

Antibiotic-producing organisms have developed several types of defensive strategies against their own product: modification or replacement of the target site for the antibiotic; inactivation or sequestration of the intracellular antibiotic; and exclusion of the drug from the cytoplasm by an efflux mechanism together with the erection of membrane permeability barriers. Several of these mechanisms can co-exist in the same strain (reviewed by Cundliffe, 1989a).

Analysis of the development of spiramycin resistance in antibiotic-producing $S$. ambofaciens led to the conclusion that at least two distinct resistance mechanisms were present. A mechanism involving ribosomal modification conferred resistance to spiramycin but also to other macrolide antibiotics. It is inducible by subinhibitory concentrations of spiramycin, can be induced in the sensitive mycelium by exogenous spiramycin added to the medium, and is probably induced by endogenous spiramycin when production begins. In the wild-type strain, this resistance seems to be induced before the detection of spiramycin in the medium, but the intracellular concentration of the antibiotic is probably sufficient for induction before the extracellular concentration is detectable. This intracellular concentration necessary for induction is probably lower than the extracellular concentration required for induction by exogenous spiramycin. Some spiramycin precursors might also induce this ribosomal resistance, but as no self-induction of the ribosomal resistance was observed for any non-producing mutant in this study, these mutants would have to be blocked before such inducing precursors were produced.

Another resistance mechanism can be detected using non-producing mutants. This resistance was not ribosomal, and conferred resistance to some but not all 
macrolide antibiotics, possibly only to the 16 -membered macrolides. This resistance was not constitutively expressed, since it appeared specifically in $72 \mathrm{~h}$ cultures. It might be regulated similarly to the production genes, because all non-producing mutants were able to cosynthesize spiramycin; in these mutants, the production genes were probably turned on at the same time as in the wild-type strain (between 36 and $48 \mathrm{~h}$ in our culture conditions). The second resistance mechanism began to be expressed at that time. Moreover, under culture conditions retarding the onset of spiramycin production in the wild-type strain, the appearance of resistance in the non-producing mutants was similarly delayed (data not shown). In some non-producing mutants, unable to cosynthesize spiramycin, and deficient in aerial mycelium formation, this resistance is also affected (data not shown). These observations could indicate that the nonribosomal resistance is turned on at the onset of production. Concerning the biochemical basis of this non-ribosomal resistance, Fierro et al. (1988) failed to detect any spiramycin modification by methylation, acetylation or adenosylation, using extract from $S$. ambofaciens. These authors found that $S$. ambofaciens had a decrease in permeability specific to spiramycin, which could be the basis for resistance in the nonproducing mutants. The resistance could in fact correspond to a mechanism of spiramycin secretion, and it could be considered as the last step of the biosynthetic pathway rather than as a resistance mechanism sensu stricto. In the wild-type strain, during the production phase, we have shown that the ribosomal resistance was active, but this non-ribosomal resistance detected in the non-producing mutants is probably also active in the wild-type strain.

The existence of at least two different resistance mechanisms is in agreement with the fact that several resistance determinants, ( $\operatorname{srm} A, \operatorname{srm} B, \operatorname{srm} C$ and $\operatorname{srm} D)$, have been cloned from $S$. ambofaciens (Richardson et al., 1987; Pernodet and others, unpublished data). Moreover, among these resistance determinants, $\operatorname{srm} A$ and possibly $s r m D$ confer resistance through ribosomal modification (Pernodet and others, unpublished data). One or both of these determinants might be responsible for the spiramycin-inducible ribosomal resistance to macrolides observed in $S$. ambofaciens. Schoner et al. (1992) have shown that $\operatorname{srm} B$ encodes a protein possessing significant sequence similarity to ATP-dependent transport proteins. This suggests that the mechanism by which $\operatorname{srm} B$ confers resistance to spiramycin involves export of the antibiotic. Thus $\operatorname{srm} B$ could be involved in the second resistance mechanism that we observed.

Mechanisms involved in resistance to the produced antibiotic have been studied for several other macrolide producers. Ribosomal modification seems to be a widespread resistance mechanism, as rRNA methyl transferases conferring resistance have been characterized in Saccharopolyspora erythraea (Skinner et al., 1983; Uchiyama \& Weisblum, 1985), Streptomyces thermotolerans (Epp et al., 1987; Zalacain \& Cundliffe, 1990) and Streptomyces mycarofaciens (Hara \& Hutchinson, 1990 ). A strain such as $S$. fradiae, the tylosin producer, can possess two different genes encoding rRNA methyl transferases: $t$ lrA, also called ermSF (Kamimiya \& Weisblum, 1988; Zalacain \& Cundliffe, 1989) and $\operatorname{tr} D$ (Zalacain \& Cundliffe, 1991). Genes which could be involved in an efflux mechanism are also present in several macrolide producers: the genes $\operatorname{tl} C$ from $S$. fradiae and car $A$ from $S$. thermotolerans encode proteins that are highly similar to that encoded by $\operatorname{srm} B$ in $S$. ambofaciens. All these proteins possess significant sequence similarity to ATP-dependent transport proteins (Rosteck et al., 1991; Schoner et al., 1992). From $S$. antibioticus, the oleandomycin producer, no resistance determinants have been cloned, but Fierro et al. (1987) reported that this strain showed a decreased permeability to oleandomycin, suggesting a role for cell permeability in self resistance. In addition, Vilches et al. (1992) reported a macrolide glycosyl transferase activity in this strain.

To conclude, like several other macrolide producers, $S$. ambofaciens possesses at least two different resistance mechanisms. The characterization of the cloned resistance determinants and the study of their regulation will be the next step in our study. The data obtained should provide helpful information for the understanding of the self-protection towards the produced antibiotic and of the control involved in the commitment to antibiotic production.

We thank J. Dondon, F.-X. Francou, P. Lacroix and A. Sabatier for kind gift of strains or material, or for communication of unpublished results. This work was supported in part by Rhône-Poulenc Rorer. M.-T.A. received a PFPI fellowship from the Ministerio de Educación y Ciencia and from the Ministère de la Recherche et de la Technologie, and a fellowship from the EEC.

\section{References}

BALTZ, R. H. \& SENo, E. T. (1988). Genetics of Streptomyces fradiae and tylosin biosynthesis. Annual Reviews in Microbiology $\mathbf{4 2}$ $547-574$.

Barthélémy, P., Autissier, D., Gerbaud, G. \& Courvalin, P. (1984). Enzymic hydrolysis of erythromycin by a strain of Escherichia coli. Journal of Antibiotics 37, 1692-1696.

Beckmann, R. J., Cox, K. \& Seno, E. T. (1989). A cluster of tylosin biosynthetic genes is interrupted by a structurally unstable segment containing four repeated sequences. In Genetics and Molecular Biology of Industrial Microorganisms, pp. 176-186. Edited by C. L. Hershberger, S. W. Queener \& G. Hegeman. Washington, DC: American Society for Microbiology.

Bibb, M. J., Freeman, R. F. \& Hopwood, D. A. (1977). Physical and genetical characterisation of a second sex factor, SCP2, for Streptomyces coelicolor A3(2). Molecular and General Genetics 154, 155-166. 
Birmingham, V. A., Cox, K. L., Larson, J. L., Fishman, S. E., Hershberger, C. L. \& Seno, E. T. (1986). Cloning and expression of a tylosin resistance gene from a tylosin-producing strain of Streptomyces fradiae. Molecular and General Genetics 204, 532-539.

CUNDLIFFE, E. (1989a). How antibiotic-producing organisms avoid suicide. Annual Reviews in Microbiology 43, 207-223.

CUNDLIFFE, E. $(1989 b)$. Methylation of RNA and resistance to antibiotics. In Microbial Resistance to Drugs, pp. 227-248. Edited by L. E. Bryan. Berlin: Springer-Verlag.

EPP, J. K., BuRgeTt, S. G. \& SCHONER, B. E. (1987). Cloning and nucleotide sequence of a carbomycin-resistance gene from Streptomyces thermotolerans. Gene 53, 73-83.

Fierro, J. F., Hardisson, C. \& Salas, J. A. (1987). Resistance to oleandomycin in Streptomyces antibioticus, the producer organism. Journal of General Microbiology 133, 1931-1939.

Fierro, J. F., Hardisson, C. \& Salas, J. A. (1988). Involvement of cell impermeability in resistance to macrolides in some producer streptomycetes. Journal of Antibiotics 41, 142-144.

FujisaWA, Y. \& WeISBlum, B. (1981). A family of r-determinants in Streptomyces ssp. that specifies inducible resistance to macrolide, lincosamide, and streptogramin type B antibiotics. Journal of Bacteriology 146, 621-631.

Gale, E. F., Cundliffe, E., Reynolds, P. E., Richmond, M. H. \& WarIng, M. J. (1981). The Molecular Basis of Antibiotic Action. London: Wiley.

HaRA, O. \& Hutchinson, C. R. (1990). Cloning of midecamycin (MLS)-resistance genes from Streptomyces mycarofaciens, Streptomyces lividans and Streptomyces coelicolor A3(2). Journal of Antibiotics 43, 977-991.

Hopwood, D. A., BibB, M. J., Chater, K. F., Kieser, T., Bruton, C. J., Kieser, H. M., Lydiate, D. J., Smith, C. P., WARD, J. M. \& SCHREMPF, H. (1985). Genetic Manipulation of Streptomyces: a Laboratory Manual. Norwich, UK: John Innes Foundation.

JENKINS, G. \& CUNDLIFFE, E. (1991). Cloning and characterization of two genes from Streptomyces lividans that confer inducible resistance to lincomycin and macrolide antibiotics. Gene 108, 55-62.

KamimiYa, S. \& Weisblum, B. (1988). Translational attenuation control of ermSF, an inducible resistance determinant encoding rRNA $N$-methyltransferase from Streptomyces fradiae. Journal of Bacteriology 170, 1800-1811.

Kuo, M.-S., Chirby, D. G., Argoudelis, A. D., Cialdella, J. I., CoATS, J. H. \& MARShaLl, V. P. (1989). Microbial glycosylation of Erythromycin A. Antimicrobial Agents and Chemotherapy 33, 2089-2091.

LeClercQ, R. \& Courvalin, P. (1991). Bacterial resistance to macrolide, lincosamide, and streptogramin antibiotics by target modification. Antimicrobial Agents and Chemotherapy 35, 1267-1272.

Marshall, V. P., Cialdella, J. I., BaczynskyJ, L., Liggett, W. F. \& JoHnson, R. A. (1989). Microbial $O$-phosphorylation of macrolide antibiotics. Journal of Antibiotics 42, 132-134.

Morgan, E. A., Gregory, S. T., Sigmund, C. D. \& Borden, A. (1988). Antibiotic resistance mutations in Escherichia coli ribosomal RNA genes and their uses. In Genetics of Translation, New Approaches, pp. 43-53. Edited by M. F. Tuite, M. Picard \& M. Bolotin-Fukuhara. Berlin: Springer-Verlag.

OMURa, S. \& TANAKa, H. (1984). Production and antimicrobial activity of macrolides. In Macrolide Antibiotics, Chemistry, Biology and Practice, pp. 3-35. Edited by S. Omura. London: Academic Press.

Pernodet, J.-L., Boccard, F., Alegre, M.-T., Blondelet-Rouault, M.-H. \& GuÉRINEAU, M. (1988). Resistance to macrolides, lincosamides and streptogramin type $B$ antibiotics due to a mutation in an rRNA operon of Streptomyces ambofaciens. The EMBO Journal 7, $277-282$.
PinNert-Sindico, S. (1954). Une nouvelle espèce de Streptomyces productrice d'antibiotiques: Streptomyces ambofaciens $\mathrm{n}$. $\mathrm{sp}$. caractères culturaux. Annales de l'Institut Pasteur (Paris) 87, 702-707.

Pridham, T. G., Anderson, P., Foley, C., Lindenfelser, L. A., Hesseltine, C. W. \& Benetdict, R. C. (1957). A selection of media for maintenance and taxonomic study of Streptomyces. Antibiotics Annual 1956-1957 947-953.

Richardson, M. A., Kuhstoss, S., Solenberg, P., Schaus, N. A. \& RAO, R. N. (1987). A new shuttle cosmid vector, pKC505, for streptomycetes: its use in the cloning of three different spiramycinresistance genes from a Streptomyces ambofaciens library. Gene 61, 231-241.

Ross, J. I., Eady, E. A., Cove, J. H., Cunliffe, W. J., Baumberg, S. \& WootTon, J.C. (1990). Inducible erythromycin resistance in staphylococci is encoded by a member of the ATP-binding transport super-gene family. Molecular Microbiology 4, 1207-1214.

Rosteck, P. R., Reynolds, P. A. \& Hershberger, C. L. (1991). Homology between proteins controlling Streptomyces fradiae tylosin resistance and ATP-binding transport. Gene 102, 27-32.

Schoner, B., Geistlich, M., Rosteck, P., JR, RaO, R. N., Seno, E., Reynolds, P., Cox, K., Burgett, S. \& Hershberger, C. (1992). Sequence similarity between macrolide-resistance determinants and ATP-binding transport proteins. Gene 115, 93-96.

SkegGS, P. A., Thompson, J. \& Cundliffe, E. (1985). Methylation of $16 \mathrm{~S}$ ribosomal RNA and resistance to aminoglycoside antibiotics in clones of Streptomyces lividans carrying DNA from Streptomyces tenjimariensis. Molecular and General Genetics 200, 415-421.

SkinNer, R., CundlifFe, E. \& Schmidt, F. J. (1983). Site of action of a ribosomal RNA methylase responsible for resistance to erythromycin and other antibiotics. Journal of Biological Chemistry 258, 12702-12706.

Smokvina, T., Francou, F. \& Luzzati, M. (1988). Genetic analysis in Streptomyces ambofaciens. Journal of General Microbiology 134, 395-402.

UChiyama, H. \& Weisblum, B. (1985). $N$-Methyl transferase of Streptomyces erythreus that confers resistance to the macrolidelincosamide-streptogramin B antibiotics: amino acid sequence and its homology to cognate $\mathrm{R}$-factor enzymes from pathogenic bacilli and cocci. Gene 38, 103-110.

Vilches, C., Hernandez, C., Mendez, C. \& Salas, J. A. (1992). Role of glycosylation and deglycosylation in biosynthesis of and resistance to oleandomycin in the producer organism, Streptomyces antibioticus. Journal of Bacteriology 174, 161-165.

WeISBLum, B. (1983). Inducible resistance to Macrolides, Lincosamides, and Streptogramin type-B antibiotics: the resistance phenotype, its biological diversity, and structural element that regulate expression. In Gene Function in Prokaryotes, pp. 91-121. Edited by J. Beckwith, J. Davies \& J. A. Gallant. Cold Spring Harbor, NY: Cold Spring Harbor Laboratory.

Wittmann, H. G., Stöffler, G., Apirion, D., Rosen, L., Tanaka, K., Tamaki, M., Takata, R., Dekio, S., Otaka, E. \& Osawa, S. (1973). Biochemical and genetic studies on two different types of erythromycin resistant mutants of Escherichia coli with altered ribosomal proteins. Molecular and General Genetics 127, 175-189.

Zalacain, M. \& Cundliffe, E. (1989). Methylation of 23S rRNA caused by $\operatorname{tr} A$ (ermSF), a tylosin resistance determinant from Streptomyces fradiae. Journal of Bacteriology 171, 42544260.

ZalaCAin, M. \& CUNDlifFe, E. (1990). Methylation of 23S ribosomal RNA due to $\operatorname{car} B$, an antibiotic-resistance determinant from the carbomycin producer, Streptomyces thermotolerans. European Journal of Biochemistry 189, 67-72.

Zalacain, M. \& Cundliffe, E. (1991). Cloning of $t$ lrD, a fourth resistance gene, from the tylosin producer Streptomyces fradiae. Gene 97, 137-142. 